Article

\title{
Fixpointed Idempotent Uninorm (Based) Logics
}

\author{
Eunsuk Yang
}

Department of Philosophy \& Institute of Critical Thinking and Writing, Colleges of Humanities \& Social Science Blvd., Chonbuk National University, Rm 417, Jeonju 54896, Korea; eunsyang@jbnu.ac.kr

Received: 8 December 2018; Accepted: 18 January 2019; Published: 20 January 2019

\begin{abstract}
Idempotent uninorms are simply defined by fixpointed negations. These uninorms, called here fixpointed idempotent uninorms, have been extensively studied because of their simplicity, whereas logics characterizing such uninorms have not. Recently, fixpointed uninorm mingle logic (fUML) was introduced, and its standard completeness, i.e., completeness on real unit interval $[0,1]$, was proved by Baldi and Ciabattoni. However, their proof is not algebraic and does not shed any light on the algebraic feature by which an idempotent uninorm is characterized, using operations defined by a fixpointed negation. To shed a light on this feature, this paper algebraically investigates logics based on fixpointed idempotent uninorms. First, several such logics are introduced as axiomatic extensions of uninorm mingle logic (UML). The algebraic structures corresponding to the systems are then defined, and the results of the associated algebraic completeness are provided. Next, standard completeness is established for the systems using an Esteva-Godo-style approach for proving standard completeness.
\end{abstract}

Keywords: substructural fuzzy logic; algebraic completeness; standard completeness; fixpoint; idempotent uninorm

\section{Introduction}

Uninorms are a special kind of associative and commutative aggregation operators allowing an identity in unit interval $[0,1]$. They were introduced by Yager and Rybalov in Reference [1], characterizing their structure along with Fodor [2]. Since then, these kinds of operators have been extensively investigated due to their special interest from a theoretical and applicative point of view. As mentioned in Reference [3], these operators are theoretically interesting because of their structure, a special combination of a t-norm and a t-conorm, and also applicatively interesting due to their practicality in applications, e.g., aggregation in general [1,4], expert systems [5], and fuzzy system modeling [6,7].

Uninorms are divided into conjunctive and disjunctive, which are used as logical connectives modeling conjunction AND and disjunction OR operators. They are also divided into left-continuous and right-continuous uninorms, where the left-continuous ones are used as logical connectives modeling implication operators. In particular, conjunctive left-continuous uninorms are very important from a logical point of view because they give rise to standard algebraic semantics, i.e., algebraic semantics on $[0,1]$, for fuzzy logics: Several uninorm (based) logics characterizing the classes of conjunctive left-continuous uninorms have recently been introduced. For instance, weakening-free uninorm (based) logics, Uninorm Logic (UL), Involutive Uninorm Logic (IUL), Uninorm Mingle Logic (UML), and Involutive Uninorm Mingle Logic (IUML) have been introduced by Metcalfe (and Montagna) [8,9] as substructural fuzzy logics, 'substructural' in the sense that they lack various structural rules such as weakening or contraction and 'fuzzy' in the sense that they are complete with respect to (w.r.t.) linearly ordered algebras or matrices. Among them, UL is the most basic uninorm logic, which is the logic of conjunctive left-continuous uninorms and their residua. 
As mentioned in Reference [3], among different classes of uninorms, the class of idempotent uninorms is particularly interesting because of its simplicity; idempotent uninorms are special combinations of minimum and maximum. Thus, idempotent uninorms have been extensively studied in many research papers [3,10-21].

This paper is a contribution to the study of substructural fuzzy logics characterized by idempotent uninorms, defined by fixpointed negations, called here fixpointed idempotent uninorms. In general, a function $n:[0,1] \rightarrow[0,1]$ is said to be a negation function if and only if (iff) it is nonincreasing and satisfies $n(0)=1$ and $n(1)=0$. This definition works on t-norms very well, but not as well on uninorms because, e.g., a nonincreasing function $n$ may not satisfy $n(1)=0$ in (conjunctive) uninorms. Thus, we henceforth call a nonincreasing function a negation function. In this paper, a negation function (briefly stated as negation) $n$ satisfying $n(n(x)) \geq x$ for all $x \in[0,1]$ is said to be a superinvolutive negation; a superinvolutive negation $n$ is called a weak negation if $n$ further satisfies $n(0)=1$ and $n(1)=0$; a weak negation $n$ is called a strong negation (or involutive negation) if $n$ further satisfies $n(n(x))=x$ for all $x \in[0,1]$. In strong negations on $[0,1], n(x)$ may be defined as $1-x$ (the so-called 'standard' negation), because strong negations on $[0,1]$ are all isomorphic (see Reference [22]). We in particular call a negation fixpointed if it has a fixpoint. By Brouwer's fixed-point theorem, every continuous negation on the $[0,1]$ interval is fixpointed.

For the past fifteen years, idempotent uninorms and, in particular, conjunctive left-continuous idempotent uninorms, have been introduced and characterized $[3,13,14,17,23]$. Furthermore, the logics based on conjunctive left-continuous idempotent uninorms have been introduced $[9,24,25]$. For instance, not only the UML and IUML systems, mentioned above, but also the fUML system (UML with fixpoint (FP) $\mathbf{t} \leftrightarrow \neg \mathbf{t}(=\mathbf{f})$ were introduced [25]. However, to our knowledge, any other satisfactory systems that are logics of conjunctive left-continuous idempotent uninorms with superinvolutive or weak negations (in place of strong ones) introduced in References [13,14], have not yet been studied.

The purpose of this paper is to introduce logics of conjunctive left-continuous idempotent uninorms with such negations and their corresponding standard algebraic semantics, i.e., algebraic semantics based on unit interval $[0,1]$. We first note the following three facts.

First, although UML was proved to be the logic of conjunctive left-continuous idempotent uninorms [9], this system is unsatisfactory in that, while a (left-continuous) idempotent uninorm is characterized by a fixpointed negation by virtue of which the uninorm is defined as a combination of minimum and maximum $[3,13,17]$, UML itself does not prove the logical principle (FP) corresponding to the fixpoint. This fact shows that 1) not UML itself but fUML has such negation, and 2) this system may be regarded as the f-free fragment of $\mathbf{U M L}$, where negation is defined as $\varphi \rightarrow \mathbf{t}$. Note that IUML is the logic of conjunctive left-continuous idempotent uninorms with any fixpointed strong negation.

Second, although the standard completeness of fUML was proved by Baldi-Ciabattoni [25], their study is, in a sense, unsatisfactory in that, while a (left-continuous) idempotent uninorm is algebraically defined, their completeness proof is not algebraic. Note that, following Metcalfe and Montagna's method [9], Baldi and Ciabattoni proof-theoretically showed the standard completeness of fUML by virtue of introducing and eliminating density rule. Their proof does not shed any light on the algebraic feature by which an idempotent uninorm is characterized using operations defined by a fixpointed negation.

Thirdly, although the standard completeness of some particular extensions of UML was algebraically proved by Wang [26,27] and Yang [28], their constructions were not established using the definitions obtained by fixpointed negations. Note that Wang [26,27] performed algebraic standard completeness for the $n$-potent uninorm and involutive uninorm logics $\mathbf{C}_{n} \mathbf{U L}$ and $\mathbf{C}_{n} \mathbf{I U L}$, for $3 \leq n$, using Jenei-Montagna-style construction. (As Wang stated, his approach does not work for $n=2$. For $\mathbf{C}_{2} \mathbf{U L}$ and $\mathbf{C}_{2} \mathbf{I U L}$, he instead mentioned Metcalfe-Montagna's standard completeness results of UML and IUML [9]). Yang [28] also established such completeness for $\mathbf{U L}_{c f r}$ (UL with compensation-free reinforcement axiom). But these approaches do not work for $\mathbf{U M L}\left(=\mathrm{C}_{2} \mathbf{U L}\right)$ and 
IUML $\left(=\mathrm{C}_{2}\right.$ IUL with FP). (For the similarities and differences between constructions of Yang and Wang's Jenei-Montagna-style, see Reference [29]).

The paper is organized as follows. In Section 2, we introduce five fixpointed idempotent uninorm (based) systems, define their corresponding algebraic structures, and prove their algebraic completeness. In Section 3, we introduce several conjunctive left-continuous fixpointed idempotent uninorms. In particular, we introduce finite matrices satisfying UML-algebras, but not having the fixpointed negation by virtue of which a conjunctive left-continuous idempotent uninorm is defined (see Example 2). In Section 4, we establish standard completeness for the systems using an Esteva-Godo-style approach for proving standard completeness of the Weak nilpotent minimum logic (WNM) and Nilpotent minimum logic (NM) systems in Reference [22].

One important feature in the Esteva and Godo's approach is that intensional conjunction and implication operators are defined by weak and strong negations. Note that intensional conjunction and implication operators for our systems are also defined by such negations. This means that one natural way to establish standard completeness for the systems is to define standard algebras by virtue of fixpointed negations. We verify this using the Esteva-Godo-style construction for proving standard completeness of the systems.

For convenience, we adopted notations and terminology similar to those in References [9,22,29-33], and we assume reader familiarity with them (together with results found therein).

\section{Fixpointed Idempotent Uninorm Logics}

\subsection{Axiomatizations}

The uninorm (based) logics introduced in Reference [9] are substructural fuzzy logics. Here, we base idempotent uninorm logics on a countable propositional language with formulas $F m$ built inductively as usual from a set of propositional variables $V A R$, binary connectives $\rightarrow, \&, \wedge, \vee$, and constants $T, \perp, \mathbf{f}, \mathbf{t}$, with defined connectives:

df1. $\neg \varphi:=\varphi \rightarrow \mathbf{f}$, and

df2. $\varphi \leftrightarrow \psi:=(\varphi \rightarrow \psi) \wedge(\psi \rightarrow \varphi)$.

We moreover define $\varphi_{\mathbf{t}}^{n}$ as $\varphi_{\mathbf{t}} \& \ldots \& \varphi_{\mathbf{t}}, n$ factors, where $\varphi_{\mathbf{t}}:=\varphi \wedge \mathbf{t}$, and Gödel negation $-\varphi$ as $\varphi \rightarrow \perp$. (Note that $\mathbf{t}$ may be defined as $\neg \mathbf{f}$.) We use the axiom systems in order to provide a consequence relation.

We start with the following axiomatization of UML, the most basic idempotent uninorm (based) logic introduced here.

Definition 1. UML consists of the following axiom schemes and rules:

A1. $\varphi \rightarrow \varphi$ (self-implication, SI)

A2. $(\varphi \wedge \psi) \rightarrow \varphi,(\varphi \wedge \psi) \rightarrow \psi(\wedge$-elimination, $\wedge$-E $)$

A3. $((\varphi \rightarrow \psi) \wedge(\varphi \rightarrow \chi)) \rightarrow(\varphi \rightarrow(\psi \wedge \chi))(\wedge$-introduction, $\wedge$-I)

A4. $\varphi \rightarrow(\varphi \vee \psi), \psi \rightarrow(\varphi \vee \psi)(\vee$-introduction, $\vee-I)$

A5. $((\varphi \rightarrow \chi) \wedge(\psi \rightarrow \chi)) \rightarrow((\varphi \vee \psi) \rightarrow \chi)(\vee$-elimination, $\vee-E)$

A6. $(\varphi \& \psi) \rightarrow(\psi \& \varphi)(\&$-commutativity, \&-C)

A7. $(\varphi \& t) \leftrightarrow \varphi$ (push and pop, PP)

A8. $\perp \rightarrow \varphi$ (ex falsum quodlibet, $E F)$

A9. $\varphi \rightarrow T$ (verum ex quolibet, $V E)$

A10. $(\varphi \rightarrow(\psi \rightarrow \chi)) \leftrightarrow((\varphi \& \psi) \rightarrow \chi)$ (residuation, $R E)$

A11. $(\varphi \rightarrow \psi) \rightarrow((\psi \rightarrow \chi) \rightarrow(\varphi \rightarrow \chi))$ (suffixing, $S F)$

A12. $(\varphi \rightarrow \psi)_{t} \vee(\psi \rightarrow \varphi)_{t}\left(t\right.$-prelinearity, $\left.P L_{t}\right)$

A13. $(\varphi \& \varphi) \leftrightarrow \varphi$ (idempotence, ID) 
$\varphi \rightarrow \psi, \varphi \vdash \psi$ (modus ponens, $m p)$

$\varphi, \psi \vdash \varphi \wedge \psi$ (adjunction, adj).

Fixpointed idempotent uninorm logics are defined by extending the UML with suitable axiom schemes as follows.

Definition 2. A logic is an axiomatic extension (extension for short) of $\boldsymbol{L}$ iff it results from $\boldsymbol{L}$ by adding axiom schemes. In particular, the following are fixpointed idempotent uninorm logics extending UML:

- $\quad$ Fixpointed uninorm mingle logic fUML is UML plus (fixpoint, FP) $t \leftrightarrow f$.

- $\quad$ Nonweak fixpointed uninorm mingle logic nwfUML is fUML plus (non-weakness, $N W)-(\neg \varphi \rightarrow \perp)$ ).

- Weak fixpointed uninorm mingle logic wfUML is fUML plus (weakness, $W$ ) $\neg \top \rightarrow \perp$.

- De Baets weak fixpointed uninorm mingle logic DwfUML is wfUML plus

(De Baets weakness1, DW1) $(\varphi \rightarrow \perp) \vee(-(\varphi \rightarrow \perp) \wedge(\varphi \rightarrow \boldsymbol{t})) \vee-(\varphi \rightarrow \boldsymbol{t})$ and

(De Baets weakness2, DW2) $(-(\varphi \rightarrow \perp) \wedge(\varphi \rightarrow \boldsymbol{t})) \rightarrow(\neg \varphi \rightarrow \boldsymbol{t})$.

- ([9]) Involutive uninorm mingle logic IUML is fUML plus (double negation elimination, DNE) $\neg \neg \varphi \rightarrow \varphi$.

Remark 1. Uninorm logic UL is UML minus (ID), and the multiplicative additive intuitionistic linear logic MAILL is UL minus $\left(P L_{t}\right)$.

For easy reference, we let Ls be the set of fixpointed idempotent uninorm logics introduced in Definition 2.

Definition 3. $L s=\{f U M L, n w f U M L, w f U M L, D w f U M L, I U M L\}$.

A theory is a set of formulas. A proof in theory $T$ over $\mathrm{L} \in \mathrm{Ls}$ is a sequence $s$ of formulas such that each element of $s$ is either an axiom of $\mathrm{L}$, a member of $T$, or is derivable from previous elements of $s$ by means of a rule of L. $T \vdash \varphi$, more exactly $T \vdash_{L} \varphi$, means that $\varphi$ is provable in $T$ w.r.t. L, i.e., there is an L-proof of $\varphi$ in $T$. A theory $T$ is inconsistent if $T \vdash \perp$; otherwise, it is consistent.

The $\mathbf{t}$-deduction theorem $\left(\mathrm{DT}_{\mathrm{t}}\right)$ for $\mathrm{L}$ is as follows:

Proposition 1. Let $T$ be a theory over $L \in$ Ls and $\varphi, \psi$ be formulas.

$$
\left(D T_{t}\right) T \cup\{\varphi\} \vdash_{L} \psi \text { iff } T \vdash_{L} \varphi_{t} \rightarrow \psi
$$

Proof. This is just the Enthymematic Deduction Theorem (see Reference [34]).

The following formulas can be proved straightforwardly.

\section{Proposition 2.}

(i) MAILL proves:

(1) $(\psi \rightarrow \chi) \rightarrow((\varphi \rightarrow \psi) \rightarrow(\varphi \rightarrow \chi))$ (prefixing, $P F)$

(2) $(\varphi \&(\psi \& \chi)) \rightarrow((\varphi \& \psi) \& \chi)$ (associativity, AS)

(3) $(\varphi \rightarrow \psi) \rightarrow(\neg \psi \rightarrow \neg \varphi)$ (contraposition, $C P$ )

(4) $\varphi \rightarrow \neg \neg \varphi$ (double negation introduction, DNI)

(5) $\neg \varphi \leftrightarrow \neg \neg \neg \varphi$

(6) $\neg(\varphi \vee \psi) \leftrightarrow(\neg \varphi \wedge \neg \psi)$ (de MorganI, DM1)

(7) $(\neg \varphi \vee \neg \psi) \rightarrow \neg(\varphi \wedge \psi)$

(8) $\neg \perp \leftrightarrow \top$

(9) $\perp \rightarrow \neg \top$ 
(ii) UL proves:

(1) $(\neg \varphi \vee \neg \psi) \leftrightarrow \neg(\varphi \wedge \psi)$ (de MorganII, DM2)

(iii) UML proves:
(1) $\neg \varphi \vee \neg \neg \varphi$ (negated excluded middle, nEM)
(2) $\neg(\varphi \wedge \neg \varphi)$ (non-contradiction, NC)
(3) $-\varphi \vee--\varphi$ (negated excluded middle,$n E M_{-}$)
(4) $-\varphi \rightarrow(-\varphi \leftrightarrow \top)$ (Gödel negation1, Gn1)
(5) $\quad--\varphi \rightarrow(-\varphi \leftrightarrow \perp)$ (Gödel negation2, Gn2)
(6) $-\varphi \rightarrow \neg \varphi$
(7) $(\neg \varphi \wedge \psi) \rightarrow(\varphi \rightarrow \psi)$
(8) $(\varphi \rightarrow \psi) \rightarrow((\varphi \rightarrow \psi) \rightarrow(\neg \varphi \vee \neg \neg \psi))$
(9) $(\varphi \rightarrow \psi) \rightarrow((\neg \varphi \vee \psi) \rightarrow(\varphi \rightarrow \psi))$
(10) $(\varphi \rightarrow \psi) \rightarrow((\varphi \vee \neg \psi) \rightarrow(\varphi \rightarrow \psi))$
(11) $\neg(\varphi \rightarrow \psi) \rightarrow((\varphi \rightarrow \psi) \rightarrow(\neg \varphi \wedge \neg \neg \psi))$
(12) $((\varphi \rightarrow \psi) \rightarrow(\neg \varphi \wedge \psi)) \rightarrow((\varphi \rightarrow \psi) \leftrightarrow(\neg \varphi \wedge \psi))$
(13) $(\varphi \& \psi) \rightarrow((\varphi \& \psi) \leftrightarrow(\varphi \vee \psi))$
(14) $\neg(\varphi \& \psi) \rightarrow((\varphi \wedge \psi) \leftrightarrow(\varphi \& \psi))$

(iv) fUML proves:
(1) $\neg \varphi, \neg(\varphi \rightarrow \psi) \vdash(\varphi \&(\varphi \rightarrow \psi)) \leftrightarrow(\varphi \wedge \psi)$
(2) $\varphi \vee \neg \varphi$ (excluded middle, EM)
(3) $\neg \varphi \leftrightarrow(\varphi \rightarrow t)$
(4) $(\varphi \rightarrow \psi) \vee((\varphi \rightarrow \psi) \rightarrow(\neg \varphi \wedge \psi))$
(5) $(\varphi \rightarrow \psi) \rightarrow(\neg \varphi \vee \psi)$
(6) $(\varphi \rightarrow \psi) \rightarrow((\varphi \rightarrow \psi) \leftrightarrow(\neg \varphi \vee \psi))$
(7) $((\varphi \& \psi) \leftrightarrow(\varphi \wedge \psi)) \vee((\varphi \& \psi) \leftrightarrow(\varphi \vee \psi))$

(v) nwflML proves:
(1) $-(\neg \top \rightarrow \perp)$
(2) $-\varphi \rightarrow(\neg \varphi \leftrightarrow \top)$
(3) $-\neg \varphi \rightarrow(\neg \varphi \leftrightarrow \perp)$

(vi) wfUML proves:
(1) $\neg \top \leftrightarrow \perp$

(vii) DwfUML proves:
(1) $(\varphi \rightarrow \perp) \rightarrow(\neg \varphi \leftrightarrow \top)$
(2) $(-(\varphi \rightarrow \perp) \wedge(\varphi \rightarrow \boldsymbol{t})) \rightarrow(\neg \varphi \leftrightarrow t)$
(3) $-(\varphi \rightarrow \boldsymbol{t}) \rightarrow(\neg \varphi \leftrightarrow \perp)$
(4) $(-(\varphi \rightarrow \neg \psi))_{t} \rightarrow((\varphi \& \psi) \leftrightarrow(\varphi \vee \psi))$

(viii) IUML proves:
(1) $\neg(\varphi \rightarrow \neg \psi) \rightarrow((\varphi \& \psi) \leftrightarrow(\varphi \vee \psi))$
(2) $\varphi \leftrightarrow \neg \neg \varphi$ (double negation, $D N$ )

Proof. Interesting cases are (iv) (4) and (5). We prove these two as examples: 
(4) $(\varphi \rightarrow \psi) \vee((\varphi \rightarrow \psi) \rightarrow(\neg \varphi \wedge \psi))$ : We first assume $\varphi \rightarrow \psi$ or $\neg(\varphi \rightarrow \psi)$ and show $\varphi \rightarrow \psi$ or $(\varphi \rightarrow \psi) \rightarrow(\neg \varphi \wedge \psi)$. For this, we assume $\neg(\varphi \rightarrow \psi)$ and show $(\varphi \rightarrow \psi) \rightarrow(\neg \varphi \wedge \psi)$. Let $\neg(\varphi \rightarrow \psi)$. Using (iii) (11) and (mp), we get $(\varphi \rightarrow \psi) \rightarrow(\neg \varphi \wedge \neg \neg \psi)$ and thus $(\varphi \rightarrow \psi) \rightarrow \neg \varphi$. Hence, we just need to show that $(\varphi \rightarrow \psi) \rightarrow \psi$. For this, we further assume either $\mathbf{t} \rightarrow \varphi$ or $\varphi \rightarrow \mathbf{t}$. Let $\mathbf{t} \rightarrow \varphi$. Using (SF) and (mp), we obtain $(\varphi \rightarrow \psi) \rightarrow(\mathbf{t} \rightarrow \psi)$ and thus $(\varphi \rightarrow \psi) \rightarrow \psi$. Let $\varphi \rightarrow \mathbf{t}$. Using the suppositions and (iv) (1), we can obtain $(\varphi \rightarrow \psi) \rightarrow \psi$ since $(\varphi \wedge \psi) \leftrightarrow \psi$. Then, from the suppositions, we have $(\varphi \rightarrow \psi) \rightarrow(\neg \varphi \wedge \psi)$ using (adj), A3, and (mp). Moreover, we can eliminate the suppositions using (iv) (2) and the $\mathbf{t}$-deduction theorem $\left(\mathrm{DT}_{\mathbf{t}}\right)$. Therefore, we obtain $(\varphi \rightarrow \psi) \vee((\varphi \rightarrow \psi) \rightarrow(\neg \varphi \wedge \psi))$, i.e., (4).

(5) $(\varphi \rightarrow \psi) \rightarrow(\neg \varphi \vee \psi)$ : We first assume either $\psi \rightarrow \mathbf{t}$ or $\mathbf{t} \rightarrow \psi$. Let $\psi \rightarrow \mathbf{t}$. Using (PF) and (mp), we obtain $(\varphi \rightarrow \psi) \rightarrow(\varphi \rightarrow \mathbf{t})$; therefore, $(\varphi \rightarrow \psi) \rightarrow(\neg \varphi \vee \psi)$ using (iv) (3) and A4. Let $\mathbf{t} \rightarrow \psi$. We further assume either $\varphi \rightarrow \mathbf{t}$ or $\mathbf{t} \rightarrow \varphi$. Let $\mathbf{t} \rightarrow \varphi$. Using (SF) and (mp), we obtain $(\varphi \rightarrow \psi) \rightarrow(\mathbf{t} \rightarrow \psi)$ and thus $(\varphi \rightarrow \psi) \rightarrow(\neg \varphi \vee \psi)$. Let $\varphi \rightarrow \mathbf{t}$. If $((\varphi \rightarrow \psi) \& \varphi) \rightarrow \mathbf{t}$, then $(\varphi \rightarrow \psi) \rightarrow \neg \varphi$ and thus $(\varphi \rightarrow \psi) \rightarrow(\neg \varphi \vee \psi)$. Let $\mathbf{t} \rightarrow((\varphi \rightarrow \psi) \& \varphi)$. Then, using (iii) (13) and (mp), we have $((\varphi \rightarrow \psi) \& \varphi) \leftrightarrow((\varphi \rightarrow \psi) \vee \varphi)$; therefore, $((\varphi \rightarrow \psi) \vee \varphi) \rightarrow \psi$ since $((\varphi \rightarrow \psi) \& \varphi) \rightarrow \psi$. Hence, we obtain $(\varphi \rightarrow \psi) \rightarrow \psi$; therefore, $(\varphi \rightarrow \psi) \rightarrow(\neg \varphi \vee \psi)$. Moreover, we can eliminate the suppositions using (iv) (2) and (DT $)$. Therefore, we obtain $(\varphi \rightarrow \psi) \rightarrow(\neg \varphi \vee \psi)$, i.e., (5).

The proofs for the other cases are left to the interested reader.

For convenience, ' $\neg,,^{\prime} \rightarrow,,^{\prime} \wedge,{ }^{\prime} `{ }^{\prime}, ' \top$, and ' $\perp$ ' are used ambiguously as propositional connectives and constants and as algebraic operators and special elements, but the context should clarify their meaning.

\subsection{Algebraic Semantics}

Suitable algebraic structures for fixpointed idempotent uninorm logics are obtained as varieties of residuated lattices as in Reference [35].

Definition 4. A pointed bounded commutative residuated lattice is a structure $(A, \top, \perp, t, f, \wedge, \vee, *, \rightarrow)$ such that:

(I) $(A, \top, \perp, \wedge, \vee)$ is a bounded lattice with top element $\top$ and bottom element $\perp$.

(II) $(A, *, t)$ is a commutative monoid.

(III) $y \leq x \rightarrow z$ iff $x * y \leq z$, for all $x, y, z \in A\left(R E^{\mathcal{A}}\right)$.

(IV) $f$ is an arbitrary element of $A$.

Note that the class of pointed bounded commutative residuated lattices characterizes the system MAILL. Thus, we henceforth call such residuated lattices MAILL-algebras.

By $x^{n}$, we denote $x * \ldots * x, n$ factors, and let $x_{t}:=x \wedge t$.

\section{Definition 5.}

(i) (UL-algebra) A UL-algebra is a MAILL-algebra satisfying: for all $x, y \in A,\left(P L_{t}{ }^{\mathcal{A}}\right) t \leq(x \rightarrow y)_{t} \vee(y \rightarrow$ $x)_{t}$. If the lattice order is linear or total, we will call it a UL-chain.

(ii) (UML-algebra) A UML-algebra is a UL-algebra satisfying: for all $x \in A,\left(I D^{\mathcal{A}}\right) x * x=x$.

Definition 6. (L-algebras) Let $\neg x:=x \rightarrow f$ and $-x:=x \rightarrow \perp$. We say the following algebras as L-algebras.

(i) A fUML-algebra is a UML-algebra satisfying:

$\left(F P^{\mathcal{A}}\right) t=f$.

(ii) An nwfUML-algebra is a fUML-algebra satisfying: for all $x \in A$, $\left(N W^{\mathcal{A}}\right) t \leq-(\neg x \rightarrow \perp)$. 
(iii) A wfUML-algebra is a fUML-algebra satisfying: $\left(W^{\mathcal{A}}\right) t \leq \neg \top \rightarrow \perp$.

(iv) A DwfUML-algebra is a wfUML-algebra satisfying: for all $x \in A$, $\left(D W 1^{\mathcal{A}}\right) t \leq(x \rightarrow \perp) \vee(-(x \rightarrow \perp) \wedge(x \rightarrow t)) \vee-(x \rightarrow t)$ and $\left(D W 2^{\mathcal{A}}\right) t \leq(-(x \rightarrow \perp) \wedge(x \rightarrow t)) \rightarrow(\neg x \rightarrow t)$.

(v) An IUML-algebra is a fUML-algebra satisfying: for all $x \in A$, $\left(D N E^{\mathcal{A}}\right) \neg \neg x=x$.

An MAILL-algebra is said to be linearly ordered if the ordering of its algebra is linear, i.e., $x \leq y$ or $y \leq x$ (equivalently, $x \wedge y=x$ or $x \wedge y=y$ ) for each pair $x, y$.

Definition 7. (Evaluation) Let $\mathcal{A}$ be an L-algebra. An $\mathcal{A}$-evaluation is a function $v: F m \rightarrow \mathcal{A}$ satisfying: $v(\varphi \rightarrow \psi)=v(\varphi) \rightarrow v(\psi), v(\varphi \wedge \psi)=v(\varphi) \wedge v(\psi), v(\varphi \vee \psi)=v(\varphi) \vee v(\psi), v(\varphi \& \psi)=v(\varphi) * v(\psi)$, $v(\top)=\top, v(\perp)=\perp, v(\boldsymbol{t})=t, v(f)=f,($ and hence $v(\neg \varphi)=\neg v(\varphi)$ and $v(-\varphi)=-v(\varphi)$ ).

Definition 8. ([30]) Let $\mathcal{A}$ be an L-algebra, $T$ be a theory, $\varphi$ be a formula, and $\mathcal{K}$ be a class of L-algebras.

(i) (Tautology) $\varphi$ is a $t$-tautology in $\mathcal{A}$, briefly an $\mathcal{A}$-tautology (or $\mathcal{A}$-valid), if $v(\varphi) \geq t$ for each $\mathcal{A}$-evaluation $v$.

(ii) (Model) An $\mathcal{A}$-evaluation $v$ is an $\mathcal{A}$-model of $T$ if $v(\varphi) \geq t$ for each $\varphi \in T$. By Mod $(T, \mathcal{A})$, we denote the class of $\mathcal{A}$-models of $T$.

(iii) (Semantic consequence) $\varphi$ is a semantic consequence of $T$ w.r.t. $\mathcal{K}$, denoted by $T \models \mathcal{K} \varphi$, if $\operatorname{Mod}(T, \mathcal{A})=\operatorname{Mod}(T \cup\{\varphi\}, \mathcal{A})$ for each $\mathcal{A} \in \mathcal{K}$.

Definition 9. (L-algebra, [30]) Let $\mathcal{A}, T$, and $\varphi$ be the same as in Definition 8. $\mathcal{A}$ is an L-algebra if whenever $\varphi$ is L-provable in any $T$ (i.e., $T \vdash_{L} \varphi, L$ an $L$ logic), it is a semantic consequence of $T$ w.r.t. $\{\mathcal{A}\}$ (i.e., $T \models_{\{\mathcal{A}\}} \varphi, \mathcal{A}$ a corresponding L-algebra). By $\operatorname{MOD}(L)$, we denote the class of L-algebras; and by $\operatorname{MOD}^{\ell}(L)$, the class of linearly ordered L-algebras. Finally, we use $T \models_{L} \varphi$ and $T \models_{L}^{\ell} \varphi$ in place of $T \models{ }_{M O D(L)} \varphi$ and $T \models{ }_{M O D^{\ell}(L)} \varphi$, respectively.

Theorem 1. (Strong completeness) Let $T$ be a theory over $L \in L s$ and $\varphi$ be a formula. $T \vdash_{L} \varphi$ iff $T \models_{L} \varphi$ iff $T \models{ }_{L}^{\ell} \varphi$.

Proof. We obtain this theorem as a corollary of Theorem 3.1.8 in Reference [36].

Remark 2. Proposition 2 (iii) (8), (9), (12)-(14) ensure that, in linearly ordered UML-algebras, the following hold:

(UML) If $x \leq y$, then $\neg x \vee y \leq x \rightarrow y \leq \neg x \vee \neg \neg y$, and if $x \rightarrow y \leq \neg x \wedge y$, then $x \rightarrow y=\neg x \wedge y$; if $x * y \leq f$, then $x \wedge y=x * y$, and if $t \leq x * y$, then $x * y=x \vee y$.

Whereas Proposition 2 (iv) (4) - (6) additionally ensure that, in linearly ordered fUML-algebras, the following hold:

(fUML) If $x \leq y$, then $x \rightarrow y=\neg x \vee y$, and otherwise, $x \rightarrow y=\neg x \wedge y$;

if $x * y \leq f$, then $x * y=x \wedge y$, and otherwise, $x * y=x \vee y$.

These two properties, (UML) and (fUML), show that the negation for fUML is stronger than that for $U M L$.

\section{Fixpointed Idempotent Uninorms}

In this section, by ' 1, ' ' 0, ' ' $e$, ' and ' $\partial$, ' we denote $T, \perp$, identity $t$, and any $f$, respectively, on real-unit interval $[0,1]$. We first define standard L-algebras and uninorms.

Definition 10. An L-algebra is standard iff its lattice reduct is $[0,1]$. 
In standard L-algebras, monoid operator $*$ is a uninorm.

Definition 11. A uninorm is a function $\circ:[0,1]^{2} \rightarrow[0,1]$ such that, for some $e \in[0,1]$ and for all $x, y, z \in[0,1]:$

(a) $x \circ y=y \circ x$ (commutativity),

(b) $x \circ(y \circ z)=(x \circ y) \circ z$ (associativity),

(c) $x \leq y$ implies $x \circ z \leq y \circ z$ (monotonicity), and

(d) $e \circ x=x$ (identity).

A uninorm becomes a t-norm when $e=1$ and t-conorm when $e=0$. A uninorm $\circ$ is called conjunctive if $0 \circ 1=0$ and disjunctive if $0 \circ 1=1$.

The left-continuity property of uninorms is important in the sense that left-continuous uninorms give rise to their residua, and these residua are implication operators. In other words, the left-continuity property plays an important role in establishing standard completeness for Ls as in t-norm-based logics such as MTL and IMTL. (cf. see References [22,37,38]). The operation $\circ$ is said to be residuated if there is a binary function $\rightarrow:[0,1]^{2} \rightarrow[0,1]$ satisfying $\left(\operatorname{RE}^{\mathcal{A}}\right)$ in Definition 4 (III). Then, given uninorm $\circ$, residuated implication $\rightarrow$ determined by $\circ$ is defined as $x \rightarrow y:=\sup \{z: x \circ z \leq y\}$ for all $x, y \in[0,1]$. Then we can show that, for any uninorm $\circ, \circ$ and its residuated implication $\rightarrow$ form a residuated pair iff $\circ$ is conjunctive and left-continuous in both arguments (cf. see Reference [39], Proposition 5.4.2).

It is clear that operator $*$ of any standard UL-algebra is a conjunctive uninorm with identity $t$ and residuum $\rightarrow$; conversely, any residuated uninorm gives rise to a UL-algebra as follows: If $\circ$ is a uninorm with residuum $\rightarrow$ and identity $e$, then for any $\partial \in[0,1],([0,1], 1,0, e, \partial, \min , \max , 0, \rightarrow)$ is a standard UL-algebra (see [9], Proposition 17).

Definition 12. An idempotent uninorm is a uninorm satisfying: for all $x \in[0,1], x=x * x$, i.e., $\left(I D^{\mathcal{A}}\right)$.

The general form of idempotent, associative and monotonic binary operators with an identity was given by Czogala and Drewniak [40]. In particular, by virtue of fixpointed negations, a detailed characterization for the cases of (conjunctive) left-continuous idempotent uninorms is given in [13] as follows.

Fact 1. ([13]) A binary operator $\circ$ is a conjunctive left-continuous idempotent uninorm with identity element $e \in(0,1]$ iff there is a super-involutive non-increasing function $n:[0,1] \rightarrow[0,1]$ with $n(e)=e$ and $n(0)=1$ such that for all $x, y \in[0,1]$ :

$$
x \circ y= \begin{cases}\min (x, y) & \text { if } y \leq n(x) \\ \max (x, y) & \text { otherwise. }\end{cases}
$$

Recall that a nonincreasing function $n$ is said to be a negation here. As the result in Fact 1 shows, an idempotent uninorm defined by virtue of a negation $n$ with fixpoint $n(e)=e$ is a special combination of minimum and maximum.

We state one further known interesting fact associated with conjunctive left-continuous fixpointed idempotent uninorms and their residua.

Fact 2. ([9]) Let $\mathcal{A}_{s}=\left([0,1], 1,0, \frac{1}{2}, \frac{1}{2}, \min , \max , \mathrm{o}_{s}, \rightarrow_{s}\right)$, where:

$$
x \circ_{s} y= \begin{cases}\min (x, y) & \text { if } x+y \leq 1, \\ \max (x, y) & \text { otherwise. }\end{cases}
$$

$\varphi$ is valid in all standard IUML-algebras iff $\varphi$ is valid in the IUML-algebra $\mathcal{A}_{s}$. 
Operator $\circ_{s}$ is an example of Fact 1 where $\circ_{s}$ is defined from the standard negation $n_{s}, n_{s}(x):=$ $1-x$, (see Reference [23]). Similarly, an example with an involutive negation is given by De Baets [13]. We call a negation $n$ satisfying $n(0)=1$ conjunctive. The following are further hitherto (un)known examples with fixpointed superinvolutive or weak negations.

\section{Example 1.}

(i) Given a conjunctive superinvolutive fixpointed negation $n_{1}$, i.e., a negation $n_{1}$ satisfying: for all $x \in[0,1]$,

(a) $n_{1}(e)=e$,

(b) $n_{1}\left(n_{1}(x)\right) \geq x$, and

(c) $n_{1}(0)=1$,

we can construct a conjunctive left-continuous idempotent uninorm $\circ_{1}$ given by, for all $x, y \in[0,1]$ :

$$
x \circ_{1} y= \begin{cases}\min (x, y) & \text { if } y \leq n_{1}(x) \\ \max (x, y) & \text { otherwise }\end{cases}
$$

(ii) Given a conjunctive superinvolutive fixpointed negation $n_{2}$ satisfying:

$$
(d) n_{2}(1) \neq 0
$$

we can construct a conjunctive left-continuous idempotent uninorm $\circ_{2}$ as in (i). We call this negation $n_{2}$ a conjunctive nonweak fixpointed negation.

(iii) Given a weak fixpointed negation $n_{3}$, i.e., a conjunctive superinvolutive fixpointed negation $n_{3}$ satisfying:

$$
(e) n_{3}(1)=0 \text {, }
$$

we can construct a conjunctive left-continuous idempotent uninorm $\mathrm{o}_{3}$ as in (i).

(iv) ([13]) Given a negation $n_{4}$ satisfying: for all $x \in[0,1]$,

$$
\text { (f) } n_{4}(x)= \begin{cases}1 & \text { if } x=0 \\ e & \text { if } 0<x \leq e \\ 0 & \text { otherwise }\end{cases}
$$

we can construct a conjunctive left-continuous idempotent uninorm $\circ_{4}$ as in (i). We call this negation $n_{4}$ De Baets weak fixpointed negation.

Proposition 3. Let $n_{1}, n_{2}, n_{3}$, and $n_{4}$ be the negations introduced in Example 1.

(i) Conditions (b) and (c) of negation $n_{1}$ are the conditions for the negation of a UML-chain.

(ii) Condition (a) of negation $n_{1}$ satisfies $\left(F P^{\mathcal{A}}\right)$, i.e., the condition for the negation of a fUML-chain, and vice versa.

(iii) Negation $n_{2}$ satisfies $\left(N W^{\mathcal{A}}\right)$, i.e., the condition for the negation of an nwfUML-chain, and vice versa.

(iv) Negation $n_{3}$ satisfies $\left(W^{\mathcal{A}}\right)$, i.e., the condition for the negation of a wfUML-chain, and vice versa.

(v) Negation $n_{4}$ satisfies $\left(D W 1^{\mathcal{A}}\right)$ and $\left(D W 2^{\mathcal{A}}\right)$, i.e., the conditions for the negation of a DwfUML-chain, and vice versa.

Proof. Proofs for (i), (ii), and (iv) are easy. We prove (iii) and (v).

For Proof (iii), we need to show that condition $(d)$ in Example 1 is equivalent to condition $\left(N W^{\mathcal{A}}\right)$ of nwfUML-chains. For the left-to-right direction, we assume $(d)$ and instead prove $-\neg x=0$. Let $x=0$. Then, since $\neg x=1$, we have $-\neg x=0$. Otherwise, by (d), we have $\neg x \neq 0$ and thus $-\neg x=0$. 
For the right-to-left direction, we assume $\left(N W^{\mathcal{A}}\right)$ and prove $(d)$. Since $-\neg 1=0$ and $-0=1$, we obtain $\neg 1 \neq 0$.

For Proof (v), we need to show that condition $(f)$ in Example 1 is equivalent to the conditions $\left(D W 1^{\mathcal{A}}\right)$ and $\left(D W 2^{\mathcal{A}}\right)$ in DwfUML-chains. For the left-to-right direction, we assume $(f)$ and prove $\left(D W 1^{\mathcal{A}}\right)$ and $\left(D W 2^{\mathcal{A}}\right)$. We first note that the conditions of $(f)$ ensure that the algebras satisfying $(f)$ are wfUML-algebras. For $\left(D W 1^{\mathcal{A}}\right)$, it suffices to show that either $x=0$, or $x \neq 0$ and $x \leq e$, or $x \not \leq e$, i.e., $e<x$. This is obvious from the conditions. For $\left(D W 2^{\mathcal{A}}\right)$, we need to show that $\min \{--x, \neg x\} \leq \neg \neg x$. If $x=0$, we have $--x=0$. If $e<x$, we also have $\neg x=0$. Let $0<x \leq e$. Then, we obtain $\neg x, \neg \neg x=e$. Therefore, the claim holds. For the right-to-left direction, we assume $\left(D W 1^{\mathcal{A}}\right)$ and $\left(D W 2^{\mathcal{A}}\right)$ and prove $(f)$. If $x=0$, obviously $\neg x=1$. Let $0<x \leq e$. Since $\left(D W 1^{\mathcal{A}}\right)$ implies $e \leq \neg x$ and $\left(D W 2^{\mathcal{A}}\right)$ implies $\neg x \leq e$, we have $\neg x=e$. Otherwise, $\left(D W 1^{\mathcal{A}}\right)$ implies $\neg x=0$. Thus, $(f)$ holds.

Remark 3. Proposition 3 and Fact 2 show that the negations $n_{1}, n_{2}, n_{3}, n_{4}$, and $n_{s}$ can be expressed as algebraic (in)equations for negations of L-chains (i.e., linearly ordered L-algebras) in Definition 6, corresponding to negation axioms in the fUML, nwfUML, wfUML, DwfUML, and IUML systems, respectively.

Note that negation $n_{1}$ is stronger than the negation in the UML system in the sense that the former kind of negation is not directly expressed in UML, while it is the case in fUML. Note also that negation $n_{4}$ is a special weak fixpointed negation introduced by De Baets.

The left continuity of a uninorm ensures that its corresponding residuated implication can be obtained. The following are the known facts associated to residuated implications.

\section{Fact 3.}

(i) [14] Consider a conjunctive left-continuous idempotent uninorm $\circ$ with a fixpointed negation $n$ satisfying $n(1)=0$. Then its residuated implication $\rightarrow$ is given by:

$$
x \rightarrow y= \begin{cases}\max (n(x), y) & \text { if } x \leq y \\ \min (n(x), y) & \text { otherwise. }\end{cases}
$$

(ii) $[14,23]$ Consider fixpointed standard negation $n_{s}$. Then, the corresponding residuated implication of the conjunctive left-continuous idempotent uninorm $\mathrm{o}_{\mathrm{s}}$ is given by:

$$
x \rightarrow_{s} y= \begin{cases}\max (1-x, y) & \text { if } x \leq y \\ \min (1-x, y) & \text { otherwise. }\end{cases}
$$

The corresponding residuated implications of the conjunctive left-continuous idempotent uninorms with the fixpointed negations in (i) to (iv) of Example 1 are obtained as in Fact 3.

Given a conjunctive superinvolutive negation $n$, i.e., a negation satisfying Conditions (b) and (c) in Example 1 (i), as a negation for UML-algebras, we may not construct a conjunctive left-continuous idempotent uninorm $\circ$ defined like $\circ_{1}$ because linearly ordered UML-algebras on $[0,1]$ satisfy (UML), but not (fUML) in general (see Remark 2). We give such an example as finite matrices.

Example 2. Consider an algebra $\mathcal{A}=\left(\left\{1, \frac{5}{6}, \frac{2}{3}, \frac{1}{2}, \frac{1}{3}, \frac{1}{6}, 0\right\}, 1,0, \frac{5}{6}, \frac{1}{6}\right.$, min, max $\left., *, \rightarrow\right)$, where: Letting $\neg x:=x \rightarrow \frac{1}{6}, t=\frac{5}{6}$, and $f=\frac{1}{6}$,

$$
x * y= \begin{cases}\min (x, y) & \text { if } x \leq \neg y \text { or } x, y \leq t \\ \max (x, y) & \text { otherwise; and }\end{cases}
$$




$$
x \rightarrow y= \begin{cases}\max (\neg x, \neg \neg y) & \text { if } x \leq y, \\ y & \text { if } x>y \text { and } x \leq t, \\ \min (\neg x, y) & \text { otherwise. }\end{cases}
$$

Namely, operations $*, \rightarrow$ can be given as follows:

\begin{tabular}{l|lllllll}
$*$ & $1^{+}$ & $\frac{5}{6}^{+}$ & $\frac{2}{3}$ & $\frac{1}{2}$ & $\frac{1}{3}$ & $\frac{1}{6}$ & 0 \\
\hline $1^{+}$ & 1 & 1 & 1 & 1 & 1 & 1 & 0 \\
$5^{+}$ & 1 & $\frac{5}{6}$ & $\frac{2}{3}$ & $\frac{1}{2}$ & $\frac{1}{3}$ & $\frac{1}{6}$ & 0 \\
$5^{+}$ & 1 & $\frac{2}{3}$ & $\frac{2}{3}$ & $\frac{1}{2}$ & $\frac{1}{3}$ & $\frac{1}{6}$ & 0 \\
$\frac{2}{3}$ & 1 & 1 & $\frac{1}{2}$ & $\frac{1}{2}$ & $\frac{1}{3}$ & $\frac{1}{6}$ & 0 \\
$\frac{1}{2}$ & 1 & $\frac{1}{2}$ & 1 & $\frac{1}{3}$ & $\frac{1}{3}$ & $\frac{1}{6}$ & 0 \\
$\frac{1}{3}$ & 1 & $\frac{1}{3}$ & $\frac{1}{3}$ & $\frac{1}{6}$ & $\frac{1}{6}$ & $\frac{1}{6}$ & 0 \\
$\frac{1}{6}$ & 1 & $\frac{1}{6}$ & $\frac{1}{6}$ & $\frac{1}{6}$ & 0 & 0 & 0 \\
0 & 0 & 0 & 0 & 0 & 0 & 0 & $\frac{1}{3}$ \\
$\rightarrow$ & 1 & $\frac{5}{6}$ & $\frac{2}{3}$ & $\frac{1}{3}$ & $\frac{1}{6}$ & 0 \\
\hline $1^{+}$ & 1 & 0 & 0 & 0 & 0 & 0 & 0 \\
$\frac{5}{6}$ & 1 & $\frac{5}{6}$ & $\frac{2}{3}$ & $\frac{1}{2}$ & $\frac{1}{3}$ & $\frac{1}{6}$ & 0 \\
$\frac{2}{3}$ & 1 & $\frac{5}{6}$ & $\frac{5}{6}$ & $\frac{1}{2}$ & $\frac{1}{3}$ & $\frac{1}{6}$ & 0 \\
$\frac{1}{2}$ & 1 & $\frac{5}{6}$ & $\frac{5}{6}$ & $\frac{5}{6}$ & $\frac{1}{3}$ & $\frac{1}{6}$ & 0 \\
$\frac{1}{3}$ & 1 & $\frac{5}{6}$ & $\frac{5}{6}$ & $\frac{5}{6}$ & $\frac{5}{6}$ & $\frac{1}{6}$ & 0 \\
$\frac{1}{6}$ & 1 & $\frac{5}{6}$ & $\frac{5}{6}$ & $\frac{5}{6}$ & $\frac{5}{6}$ & $\frac{5}{6}$ & 0 \\
0 & 1 & 1 & 1 & 1 & 1 & 1 & 1
\end{tabular}

In each matrix for $*$ and $\rightarrow$, superscript + indicates the designated values. (Note that $\neg 1=0$, $\neg \frac{5}{6}=\frac{1}{6}, \neg \frac{2}{3}=\frac{1}{6}, \neg \frac{1}{2}=\frac{1}{6}, \neg \frac{1}{3}=\frac{1}{6}, \neg \frac{1}{6}=\frac{5}{6}$, and $\neg 0=1$.)

The axioms of UML are $\mathcal{A}$-tautologies for all assignments of values to the variables, and the rules preserve this property. This ensures soundness, i.e., if $\vdash_{\mathrm{UML}} \varphi$, then $\models_{\mathcal{A}} \varphi$. It means that this algebra satisfies (UML). However, this algebra does not satisfy (fUML). We verify this by showing that the algebraic counterparts of (iv) (4) and (5) in Proposition 2 do not hold in the matrix for $\rightarrow$.

First, consider the case $x=\frac{2}{3}$ and $y=\frac{1}{2}$. We show that $\frac{2}{3} \rightarrow \frac{1}{2} \leq \leq \min \left\{\neg \frac{2}{3}, \frac{1}{2}\right\}$. Note that $\frac{2}{3} \rightarrow \frac{1}{2}=\frac{1}{2}$ and $\min \left\{\neg \frac{2}{3}, \frac{1}{2}\right\}=\min \left\{\frac{1}{6}, \frac{1}{2}\right\}=\frac{1}{6}$. Thus, $\frac{2}{3} \rightarrow \frac{1}{2}=\frac{1}{2}>\frac{1}{6}=\min \left\{\neg \frac{2}{3}, \frac{1}{2}\right\}$. Next consider the case $x=\frac{2}{3}$ and $y=\frac{2}{3}$. We show that $\frac{2}{3} \rightarrow \frac{2}{3} \leq \max \left\{\neg \frac{2}{3}, \frac{2}{3}\right\}$. Note that $\frac{2}{3} \rightarrow \frac{2}{3}=\frac{5}{6}$ and $\max \left\{\neg \frac{2}{3}, \frac{2}{3}\right\}=\max \left\{\frac{1}{6}, \frac{2}{3}\right\}=\frac{2}{3}$. Thus, $\frac{2}{3} \rightarrow \frac{2}{3}=\frac{5}{6}>\frac{2}{3}=\max \left\{\neg \frac{2}{3}, \frac{2}{3}\right\}$. These two examples show that the algebraic counterparts of (iv) (4) and (5), respectively, in Proposition 2 do not hold in this matrix. Therefore, the definition of $\rightarrow$ for (fUML) does not hold in the matrix.

\section{Standard Completeness}

In this section, we provide standard completeness results for Ls using the Esteva-Godo-style construction in Reference [22]. We first note that given a negation $n_{i}, i \in\{1,2,3,4\}$, in Example 1, we can define a residuated pair as follows.

\section{Proposition 4.}

(i) Let $n_{i} \in\left\{n_{1}, n_{2}, n_{3}, n_{4}\right\}$, where $n_{i}$ is the corresponding negation defined in Example 1. Given a negation $n_{i}$ on $[0,1]$, we can define a residuated pair of operations $\circ_{n_{i}}, \rightarrow_{n_{i}}$ such that $\left([0,1], \circ_{n_{i}}, \rightarrow_{n_{i}}, 1,0, e, e\right)$ is a corresponding L-chain with negation $n_{i}$.

(ii) For the standard negation $n_{s}$, i.e., $n_{s}(x)=1-x$ for all $x \in[0,1]$, we can define a residuated pair of operations $\circ_{n_{s}}, \rightarrow_{n_{s}}$ such that $\left([0,1], \circ_{n_{s}}, \rightarrow_{n_{s}}, 1,0, \frac{1}{2}, \frac{1}{2}\right)$ is the IUML-chain with negation $n_{s}$.

Proof. (i) Let $n_{i}$ be $n_{1}$ in Example 1. We first note that given a conjunctive super-involutive fixpointed negation $n_{1}$ on $[0,1]$, a uninorm $\circ_{n_{1}}$ for fUML is defined as in (i) of Example 1. Then, we can easily prove that: 
(a) $\circ_{n_{1}}$ has residuum defined by

$$
x \rightarrow_{n_{1}} y= \begin{cases}\max \left(n_{1}(x), y\right) & \text { if } x \leq y \\ \min \left(n_{1}(x), y\right) & \text { otherwise }\end{cases}
$$

(b) $\left([0,1], \circ_{n_{1}}, \rightarrow_{n_{1}}, 1,0, e, e\right)$ is a fUML-algebra, and

(c) the corresponding negation is $n_{1}$.

Since the axioms of fUML are valid in an fUML-algebra, this algebra is an fUML-algebra as well. The proofs for the other cases are analogous.

(ii) The proof for the case of standard negation is analogous to that of the negations in (i), i.e., given standard fixpointed negation $n_{s}$ on $[0,1]$, uninorm $\circ_{n_{s}}$ for IUML is defined as in Fact 2 . Then, the corresponding residuated implication $\rightarrow_{n_{s}}$ is given as in (ii) of Fact 3 . Furthermore, we can easily prove that $\left([0,1], \circ_{n_{s}}, \rightarrow_{n_{s}}, 1,0, \frac{1}{2}, \frac{1}{2}\right)$ is an IUML-algebra, and corresponding negation $n_{\mathcal{S}}$ is the standard one.

Fact 4. ([22]) Strong negations on $[0,1]$ are all isomorphic.

Then, due to Fact 4, we get the following result.

Proposition 5. Strong negations on $[0,1]$ are all isomorphic so that IUML-chains defined by IUML-uninorms are.

Theorem 2. (Weak standard completeness)

(i) For $L \in\{f U M L, n w f U M L, w f U M L, D w f U M L\}, \vdash_{L} \varphi$ iff $\varphi$ is a tautology in all standard $L$-algebras.

(ii) $\vdash_{\text {IUML }} \varphi$ iff $\varphi$ is a tautology in all standard IUML-algebras iff $\varphi$ is a tautology in the standard IUML-algebra $\left([0,1], \circ_{n_{s}}, \rightarrow_{n_{s}}, 1,0, \frac{1}{2}, \frac{1}{2}\right)$.

Proof. Each proof of (i) and (ii) is analogous to that of Theorems 3 and 4, respectively, in Reference [22].

(i) Soundness is obvious. For completeness, let $\forall_{L} \varphi$. We show that there is an L-chain $C$ and an evaluation $v$ on $C$ such that $v(\varphi)<e$. We prove the case $n_{1 C}$. Let $n_{1 C}$ be the negation in $C$; take $X$ as the finite subset of $C$ consisting of all the values $v(\psi), n_{1 C}(v(\psi))$, and $n_{1 C}\left(n_{1 C}(v(\psi))\right)$ for all subformulas $\psi$ of $\varphi$, plus $0_{C}, 1_{C}$, and $e_{C}\left(=\partial_{C}\right)$. (Note that Proposition 2 (i) (5) ensures $n_{1 C}\left(n_{1 C}\left(n_{1 C}(v(\psi))\right)\right)=n_{1 C}(v(\psi))$. Thus, we do not need to consider $n_{1 C}\left(n_{1 C}\left(n_{1 C}(v(\psi))\right)\right)$.) Let

$$
X=\left\{0_{C}=a_{0}<a_{1}<\cdots<e_{C}=\partial_{C}<\cdots<a_{m}=1_{C}\right\},
$$

where $e_{C}=a_{\frac{m}{2}}$ if $m$ is even and otherwise, $e_{C}=a_{\frac{m+1}{2}}$. Let $f: X \rightarrow[0,1]$ be an ordered mapping $(x<y$ implies $f(x)<f(y))$ such that $f\left(a_{i}\right)=\frac{i}{m}$. Define on $[0,1]$ the negation function $n_{1}$ as follows:

$$
n_{1}(x)= \begin{cases}1-x & \text { if } m \text { is even and } x \in\left\{\frac{i}{m}: 0 \leq i \leq m\right\}, \\ \frac{m-i-1}{m} & \text { if } m \text { is even and } x \in\left(\frac{i}{m}, \frac{i+1}{m}\right), \\ 1 & \text { if } m \text { is odd and } x=0, \\ 1-\left(x-\frac{1}{m}\right) & \text { if } m \text { is odd and } x \in\left\{\frac{i}{m}: 0<i \leq m\right\}, \\ \frac{m-i}{m} & \text { otherwise. }\end{cases}
$$

It is clear that $n_{1}$ is a conjunctive superinvolutive fixpointed negation on $[0,1]$ and $f$ is a morphism w.r.t. minimum, maximum, implication, and negation. Note that we take $e=f\left(e_{C}\right)$. Thus, by defining the evaluation $v^{\prime}(p)=f(v(p))$ for any propositional variable $p$ occurring in $\varphi$, we get $v^{\prime}(\varphi)=f(v(\varphi))<e$, as desired.

The proofs for the other cases are analogous. Here, we just define $n_{2}, n_{3}$, and $n_{4}$. 
For $e_{C}$ such that $e_{C}=a_{\frac{m}{2}}$ if $m$ is even and otherwise $e_{C}=a_{\frac{m+1}{2}}$, define on $[0,1]$ the nonweak fixpointed negation function $n_{2}$ as follows: Taking $e=f\left(e_{C}\right)$, and letting $x^{-}$be $x-\frac{1}{m}$,

$$
n_{2}(x)= \begin{cases}e^{-} & \text {if } e<x, \\ 1 & \text { if } x \leq e^{-}, \\ e & \text { otherwise, i.e., if } e^{-}<x \leq e .\end{cases}
$$

For $e_{C}$ such that $e_{C}=a_{\frac{m}{2}}$ if $m$ is even and otherwise, $e_{C}=a_{\frac{m+1}{2}}$, define on $[0,1]$ the weak fixpointed negation function $n_{3}$ as follows: Taking $e=f\left(e_{C}\right)$, and letting $x^{-}$be $x-\frac{1}{m}$,

$$
n_{3}(x)= \begin{cases}1-x & \text { if } x \in\left\{\frac{i}{m}: 0 \leq i \leq m\right\} \text { and } x \neq e, \\ e & \text { if } e^{-}<x \leq e, \\ \frac{m-i-1}{m} & \text { otherwise. }\end{cases}
$$

For $e_{C}$ such that $e_{C}=a_{\frac{m}{2}}$ if $m$ is even and otherwise, $e_{C}=a_{\frac{m+1}{2}}$, define on $[0,1]$ the weak fixpointed negation function $n_{4}$ as follows: Taking $e=f\left(e_{C}\right)$,

$$
n_{4}(x)= \begin{cases}e & \text { if } 0<x \leq e \\ 0 & \text { if } e<x \\ 1 & \text { otherwise }\end{cases}
$$

The proof of the remaining is almost the same as in the case $n_{1 C}$.

(ii) Soundness is obvious. For completeness, let $\forall$ IUML $\varphi$. Then, there is an IUML-chain $C$ and an evaluation $v$ on $C$ such that $v(\varphi)<e$. Let $n_{C}$ be a strong negation in $C$; take $X$ as the finite subset of $C$, whose number of elements being odd, consisting of all the values $v(\psi)$ and $n_{C}(v(\psi))$, i.e., the strong negation of $v(\psi)$, for all subformulas $\psi$ of $\varphi$ together with $0_{C}, 1_{C}$, and $e_{C}\left(=\partial_{C}\right)$. Suppose that $X$ has $m+1$ elements and let $X=\left\{0_{C}=a_{0}<a_{1}<\cdots<e_{C}=\partial_{C}<\cdots<a_{m}=1_{C}\right\}$, where $e_{C}=a_{\frac{m}{2}}$ and $m$ is even. Now let $f\left(a_{i}\right)=\frac{i}{m}, 0 \leq i \leq m$. It is clear that $f$, as a mapping from $X$ to the set $\left\{\frac{i}{m}: 0 \leq i \leq m\right\}$, is a morphism w.r.t. minimum, maximum, implication, and negation; moreover, we take $e=f\left(e_{C}\right)$. Thus, defining $v^{\prime}(p)=f(v(p))$ for any propositional variable $p$ occurring in $\varphi$, we get $v^{\prime}(\varphi)=f(v(\varphi))<e$, as required. Thus, $\vdash_{\text {IUML }} \varphi$ iff $\varphi$ is a tautology in all standard IUML-algebras.

Finally note that Proposition 5 ensures that $\varphi$ is a tautology in all standard IUML-algebras iff $\varphi$ is a tautology in the standard IUML-algebra $\left([0,1], \circ_{n_{s}}, \rightarrow_{n_{s}}, 1,0, \frac{1}{2}, \frac{1}{2}\right)$.

Furthermore, we can show the strong standard completeness for Ls.

Theorem 3. (Strong standard completeness) Let $T$ be a theory over $L \in L s$, and $\varphi$ a formula. $T \vdash_{L} \varphi$ iff $\varphi$ is true in each standard $\mathbf{L}$-model of $T$.

Proof. The left-to-right direction follows from the definition. For the right-to-left direction, let $\varphi$ be a formula such that $T \nvdash_{L} \varphi, \mathcal{A}$ be a linearly ordered L-algebra, and $v$ an evaluation in $\mathcal{A}$ such that $v(\psi) \geq t$ for all $\psi \in T$ and $v(\varphi)<t$. Let $f$ be the embedding of $\mathcal{A}$ into the standard L-algebra as in Theorem 2. Then, $f \circ v$ is an evaluation into the standard L-algebra such that $f \circ v(\psi) \geq e$ and yet $f \circ v(\varphi)<e$.

\section{Concluding Remarks}

In this paper, we first introduced several fixpointed idempotent uninorm (based) systems. After defining their corresponding algebraic structures, we briefly considered algebraic completeness results for the systems. Finally, we proved their algebraic standard completeness using the Esteva-Godo-style construction for proving standard completeness. 
Note that, while algebraic completeness of some predicate uninorm logics is proved in Reference [41], such completeness for the predicate logics corresponding to the propositional logics introduced in Section 2 is not yet established. Note also that, while standard completeness for some uninorm-based logics has been established using Jenei-Montagna-style construction (see References [26-28]), such completeness for fixpointed idempotent uninorm-based systems has not been done using this construction. These are problems left in this paper.

Funding: This work was supported by the Ministry of Education of the Republic of Korea and the National Research Foundation of Korea (NRF-2016S1A5A8018255).

Conflicts of Interest: The author declares no conflict of interest.

\section{References}

1. Yager, R.R.; Rybalov, A. Uninorm aggregation operators. Fuzzy Sets Syst. 1996, 80, 111-120. [CrossRef]

2. Fodor, J.C.; Yager, R.R.; Rybalov, A. Structure of uninorms. Int. J. Uncertain. Fuzziness Knowl. Based Syst. 1997, 6, 411-427. [CrossRef]

3. Ruiz-Aguilera, D.; Torrens, J.; De Beats, B.; Fodor, J. Some remarks on the characterization of idempotent uninorms. In IPMU 2010: Computational Intelligence for Knowledge-Based Systems; Springer: Heidelberg, Germany, 2010; pp. 425-434.

4. Calvo, T.; Mayor, G.; Mesiar, R. (Eds.) Aggregation Operators: New Trends and Applications, Studies in Fuzziness and Soft Computing; Physica-Verlag: Heidelberg, Germany, 2002; Volume 97.

5. De Baets, B.; Fodor, J. Van Melle's combining function in MYCIN is a representable uninorm: An alternative proof. Fuzzy Sets Syst. 1999, 104, 133-136. [CrossRef]

6. Yager, R.R. Uninorms in fuzzy systems modeling. Fuzzy Sets Syst. 2001, 122, 167-175. [CrossRef]

7. Yager, R.R.; Kreinovich, V. Universal approximation theorem for uninorm-based fuzzy systems modelling. Fuzzy Sets Syst. 2003, 140, 331-339. [CrossRef]

8. Metcalfe, G. Uninorm Based Logics. In Proceedings of EUROFUSE; Exit Press: Warsaw, Poland, 2004; pp. 85-99.

9. Metcalfe, G.; Montagna, F. Substructural Fuzzy Logics. J. Symb. Logic 2007, 72, 834-864. [CrossRef]

10. Bustince, H.; Beliakov, G.; Dimuro, G.P.; Bedregal, B.; Mesiar, R. On the definition of penalty functions in data aggregation. Fuzzy Sets Syst. 2017, 323, 1-18. [CrossRef]

11. Çaylı, G.D.; Drygaś, P. Some properties of idempotent uninorms on a special class of bounded lattices. Inf. Sci. 2018, 422, 352-363. [CrossRef]

12. Couceiro, M.; Devillet, J.; Marichal, J. Characterizations of idempotent discrete uninorms. Fuzzy Sets Syst. 2018, 334, 60-72. [CrossRef]

13. De Baets, B. Idempotent uninorms. Eur. J. Oper. Res. 1999, 118, 631-642. [CrossRef]

14. De Baets, B.; Fodor, J. Residual operators of uninorms. Soft Comput. 1999, 3, 89-100. [CrossRef]

15. De Baets, B.; Fodor, J.; Ruiz-Aguilera, D.; Torrens, J. Idempotent uninorms on finite ordinal scales. Int. J. Uncertain. Fuzziness Knowl. Based Syst. 2009, 17, 1-14. [CrossRef]

16. Drygaś, P. Some remarks about idempotent uninorms on complete lattice. In Advances in Fuzzy Logic and Technology 2017; Kacprzyk, J., Szmidt, E., Zadroz̄ny, S., Atanassov, K., Krawczak, M., Eds.; Springer: Cham, Switzerland, 2018; Volume 641, pp. 648-657.

17. Martin, J.; Mayor, G.; Torrens, G. On locally internal monotonic operations. Fuzzy Sets Syst. 2003, 137, $27-42$. [CrossRef]

18. Massant, S.; Ruiz-Aguilera, D.; Torrens, J. Characterization of a class of fuzzy implication functions satisfying the law of importation with respect to a fixed uninorm-Part II. IEEE Trans. Fuzz Syst. 2018, 26, 1995-2003. [CrossRef]

19. Ruiz-Aguilera, D.; Torrens, J. Distributive idempotent uninorms. Int. J. Uncertain. Fuzziness Knowl. Based Syst. 2003, 11, 413-428. [CrossRef]

20. Ruiz-Aguilera, D.; Torrens, J. Residual implications and co-implications from idempotent uninorms. Kybernetika 2004, 40, 21-38.

21. Zhan, H.; Liu, H. The cross-migrative property for uninorms. Aequ. Math. 2016, 90, 1219-1239. [CrossRef] 
22. Esteva, F.; Godo, L. Monoidal t-norm based logic: Towards a logic for left-continuous t-norms. Fuzzy Sets Syst. 2001, 124, 271-288. [CrossRef]

23. Klement, E.P.; Mesiar, R.; Pap, E. Triangular Norms; Kluwer: Dordrecht, The Netherlands, 2000.

24. Baldi, P. A note on standard completeness for some extensions of uninorm logic. Soft Comput. 2014, 18, 1463-1470. [CrossRef]

25. Baldi, P.; Ciabattoni, A. Standard completeness for uninorm-based logics. In IEEE International Symposium on Multiple-Valued Logic; IEEE: New York, NY, USA, 2015; pp. 78-83.

26. Wang, S. Uninorm logic with the n-potency axiom. Fuzzy Sets Syst. 2012, 205, 116-126. [CrossRef]

27. Wang, S. Involutive uninorm logic with the n-potency axiom. Fuzzy Sets Syst. 2013, 218, 1-23. [CrossRef]

28. Yang, E. An axiomatic extension of the uninorm logic revisited. Korean J. Logic 2014, 17, 323-348.

29. Yang, E. Basic substructural core fuzzy logics and their extensions: Mianorm-based logics. Fuzzy Sets Syst. 2016, 301, 1-18. [CrossRef]

30. Cintula, P. Weakly Implicative (Fuzzy) Logics I: Basic properties. Arch. Math. Logic 2006, 45, 673-704. [CrossRef]

31. Esteva, F.; Godo, L.; Hájek, P.; Navara, M. Residuated fuzzy logics with an involutive logics. Arch. Math. Logic 2000, 39, 103-124. [CrossRef]

32. Hájek, P. Metamathematics of Fuzzy Logic; Kluwer: Amsterdam, The Netherlands, 1998.

33. Yang, E. Weakening-free, non-associative fuzzy logics: Micanorm-based logics. Fuzzy Sets Syst. 2015, 276, 43-58. [CrossRef]

34. Meyer, R.K.; Dunn, J.M.; Leblanc, H. Completeness of relevant quantification theories. Notre Dame J. Form. Logic 1976, 15, 97-121. [CrossRef]

35. Galatos, N.; Jipsen, P.; Kowalski, T.; Ono, H. Residuated Lattices: An Algebraic Glimpse at Substructural Logics; Elsevier: Amsterdam, The Netherlands, 2007.

36. Cintula, P.; Noguera, C. A general framework for mathematical fuzzy logic. In Handbook of Mathematical Fuzzy Logic; Cintula, P., Hájek, P., Noguera, C., Eds.; College Publications: London, UK, 2011; Volume 1, pp. 103-207.

37. Esteva, F.; Gispert, L.; Godo, L.; Montagna, F. On the standard and rational completeness of some axiomatic extensions of the monoidal t-norm logic. Stud. Log. 2002, 71, 393-420. [CrossRef]

38. Jenei, S.; Montagna, F. A Proof of Standard completeness for Esteva and Godo's Logic MTL. Stud. Log. 2002, 70, 183-192. [CrossRef]

39. Gottwald, S. A Treatise on Many-Valued Logics; Research Studies Press LTD.: Baldock, UK, 2001.

40. Czogala, E.; Drewniak, J. Associative monotonic operations in fuzzy set theory. Fuzzy Sets Syst. 1984, 12, $249-269$. [CrossRef]

41. Metcalfe, G.; Olivetti, N.; Gabbay, D. Proof Theory for Fuzzy Logics; Springer: Heidelberg, Germany, 2009. 